\title{
A method for simultaneous linear optics and coupling correction for storage rings with turn-by-turn beam position monitor data
}

\author{
Xi Yang
}

Brookhaven National Laboratory, Upton, Long Island, NY 11973, USA

Xiaobiao Huang

SLAC National Accelerator Laboratory, 2575 Sand Hill Road, Menlo Park, CA 94025

\section{Abstract}

We propose a method to simultaneously correct linear optics errors and linear coupling for storage rings using turn-by-turn ( $\mathrm{TbT}$ ) beam position monitor (BPM) data. The independent component analysis (ICA) method is used to isolate the betatron normal modes from the measured TbT BPM data. The betatron amplitudes and phase advances of the projections of the normal modes on the horizontal and vertical planes are then extracted, which, combined with dispersion measurement, are used to fit the lattice model. The fitting results are used for lattice correction. The method has been successfully demonstrated on the NSLS-II storage ring.

9 Keywords: optics correction, turn-by-turn, BPM

Email address: xiahuang@slac.stanford.edu (Xiaobiao Huang)

Preprint submitted to Nuclear Inst. and Methods in Physics Research, A April 26, 2016 


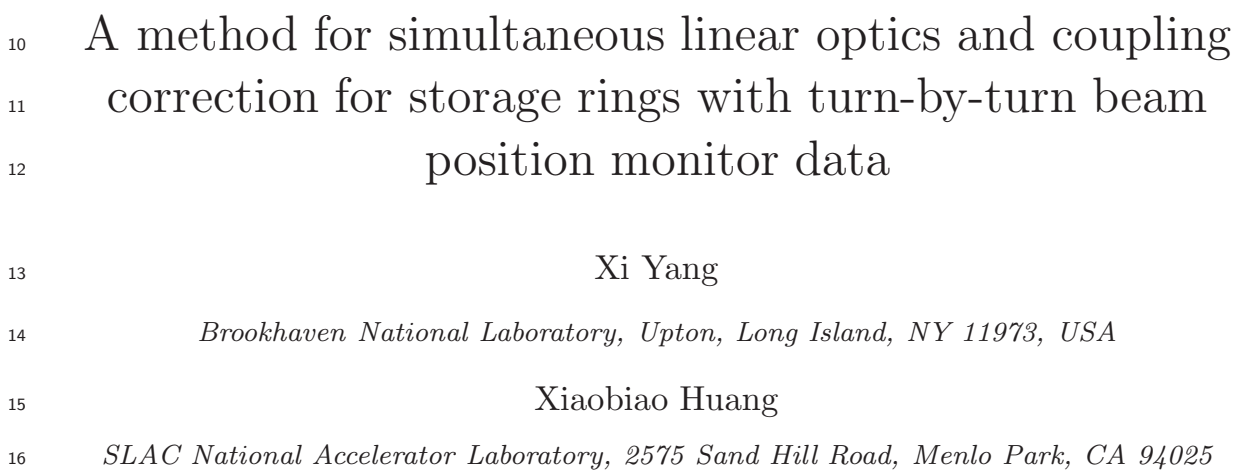

17 1. Introduction 
to be controlled. Linear coupling can be caused by skew quadrupole components of magnets through magnet errors, rolls of quadrupoles, and vertical orbit offset in sextupole magnets. Spurious vertical dispersion can be caused by vertical steering magnets and coupling of the horizontal dispersion through skew quadrupole components in dispersive regions. Both linear coupling and spurious vertical dispersion contribute to the vertical emittance and both can be corrected with skew quadrupoles. The reduction of vertical emittance through linear coupling and spurious vertical dispersion correction is often referred to as "coupling correction".

Linear optics and coupling correction for storage rings is typically done with orbit response matrix based methods (e.g., LOCO (linear optics from closed orbit) [1], used in this paper). By fitting quadrupole and skew quadrupole variables in the lattice model to the measured orbit response matrix and dispersion data, LOCO finds a set of magnet errors that can give rise to the observed lattice errors. Correcting the magnet errors in the machine then leads to improved linear optics and reduced coupling error.

In recent years turn-by-turn (TbT) BPMs have become widely used in storage rings. TbT BPMs not only detect the closed orbit, but also the orbit of a beam in coherent oscillation. From the latter betatron amplitudes and phase advances can be derived [2-4], which in turn can be used for optics correction [48]. TbT BPM data also contain linear coupling information and can be used for coupling correction. Methods based on the correction of global and/or local linear coupling resonance driving term were previously proposed or carried out by several authors [9-13]. Typically these methods require linear optics correction beforehand in order to obtain an accurate optics model as needed for coupling correction. Ref. [14] proposed a method that could be used to simultaneously correct linear optics and coupling. One disadvantage of this method is that the BPM errors of the first two BPMs propagate downstream and may affect the fitting results. The extended section by section technique (SBST) developed at LHC can be used for optics and coupling correction [15]. The extended SBST method has not been used on storage ring light sources, probably because of its 
relative complexity as compared to the LOCO method.

In this paper we propose and experimentally demonstrate a new method to simultaneously correct linear optics and coupling. The independent component analysis (ICA) method is first applied to extract the amplitudes and phases of the projection of the normal modes on the horizontal and vertical BPMs [4], which are then compared to their model generated counterparts in fitting. The fitting scheme is similar to LOCO. Since closed orbit response and coherent orbit oscillation sample the optics and coupling errors of the machine in a similar fashion, it is expected the performance of this method would be similar to that of LOCO. However, the TbT BPM data based method has a great advantage in that data taking is significantly faster than LOCO. The time for taking orbit response matrix data may vary from 10-100 minutes for different machines, while TbT BPM data taking takes only a few seconds. Simulation results for our new method were previously reported in Ref. [16].

In the following we first describe the method in section 2. A discussion of simulation results is in section 3. Experimental results on the National Synchrotron Light Source-II (NSLS-II) storage ring are presented in section 4. Conclusion is given in section 5 .

\section{Optics and coupling correction with ICA}

Betatron motion with linear coupling can be decoupled into two normal modes $[17,18]$. In general, the beam motion observed by a BPM on any of the two transverse planes has components of both normal modes. Normally the two modes have different betatron tunes and hence can be separated with the ICA method when TbT BPM data from BPMs around the ring are analyzed together [4]. In the ICA process, BPM noise is reduced and other components of beam motion, such as synchrotron motion and nonlinear resonance terms, are isolated from the betatron motion. Therefore, the resulting betatron components have high accuracy.

Each betatron normal mode corresponds to two orthogonal ICA modes. The betatron components on each BPM consist of four ICA modes, which can be 
expressed as

$$
\begin{aligned}
& x_{n}=A \cos \Psi_{1 n}-B \sin \Psi_{1 n}+c \cos \Psi_{2 n}-d \sin \Psi_{2 n}, \\
& y_{n}=a \cos \Psi_{1 n}-b \sin \Psi_{1 n}+C \cos \Psi_{2 n}-D \sin \Psi_{2 n},
\end{aligned}
$$

96 where $x_{n}$ and $y_{n}$ are observed beam positions on the horizontal and vertical

${ }_{97}$ planes at the $n$ 'th turn, respectively, $\Psi_{1 n, 2 n}=2 \pi \nu_{1,2} n+\psi_{1,2}$, and $\nu_{1,2}$ and ${ }_{98} \psi_{1,2}$ are the tunes and initial phases of the normal modes. The initial phases ${ }_{99} \psi_{1,2}$ are equal for all BPMs. Typically in a storage ring the linear coupling is 100 weak, in which case the observed $x$-motion is dominated by one normal mode and the $y$-motion by the other. For each transverse plane we call the dominant mode the primary mode and the other mode the secondary mode. The tunes of the primary modes are close to the uncoupled tunes for the corresponding planes. For the convenience of discussion, we refer the horizontal primary mode as normal mode 1 and the vertical primary mode as normal mode 2 .

The linear coupled motion of betatron coordinates $X=\left(x, x^{\prime}, y, y^{\prime}\right)^{T}$ at any location of the ring can be predicted with the one-turn transfer matrix $\mathbf{T}$. Diagonalizing the transfer matrix, one can relate betatron coordinates to normal mode coordinates

$$
\Theta=\left(\begin{array}{c}
\sqrt{2 J_{1}} \cos \Phi_{1} \\
-\sqrt{2 J_{1}} \sin \Phi_{1} \\
\sqrt{2 J_{2}} \cos \Phi_{2} \\
-\sqrt{2 J_{2}} \sin \Phi_{2}
\end{array}\right)
$$

via a transformation $\mathbf{X}=\mathbf{P} \Theta$, where $J_{1,2}$ and $\Phi_{1,2}$ are the action and phase variables for the two normal modes, respectively [19]. In particular, the position coordinates $x$ and $y$ are given by

$$
\begin{aligned}
& x=p_{11} \sqrt{2 J_{1}} \cos \Phi_{1}+\sqrt{2 J_{2}}\left(p_{13} \cos \Phi_{2}-p_{14} \sin \Phi_{2}\right), \\
& y=\sqrt{2 J_{1}}\left(p_{31} \cos \Phi_{1}-p_{32} \sin \Phi_{1}\right)+p_{33} \sqrt{2 J_{2}} \cos \Phi_{2},
\end{aligned}
$$

${ }_{13}$ where the $p_{i j}$ coefficients are elements of matrix $\mathbf{P}$ and by choice of the initial values of phase variables $\Phi_{1,2}$, we have $p_{12}=p_{34}=0$ [19]. Not considering 
damping of the coherent motion (e.g., due to decoherence), the action variables are constants of motion. The phase variables $\Phi_{1,2}$ advances from one location to another and the phase advances for a full turn are $2 \pi \nu_{1,2}$.

Clearly the measured beam motion in Eq. (1) and the model predicted motion in Eq. (3) represent the same physical process and are separated in the same form. The amplitudes and phase advances of the two normal modes on the two transverse planes in the two equations should be equal. Equating the amplitudes, we obtain

$$
\begin{aligned}
\sqrt{A^{2}+B^{2}} & =\sqrt{2 J_{1}} p_{11}, \\
\sqrt{c^{2}+d^{2}} & =\sqrt{2 J_{2}} \sqrt{p_{13}^{2}+p_{14}^{2}} \\
\sqrt{C^{2}+D^{2}} & =\sqrt{2 J_{2}} p_{33} \\
\sqrt{a^{2}+b^{2}} & =\sqrt{2 J_{1}} \sqrt{p_{31}^{2}+p_{32}^{2}} .
\end{aligned}
$$

The $J_{1,2}$ constants can be calculated by averaging the values derived from the amplitudes of the primary modes, i.e., using Eqs. (4) and (6). Aside from constant initial phase, the phase advances can also be equated, leading to

$$
\begin{aligned}
\tan ^{-1} \frac{B}{A} & =\operatorname{Mod}_{2 \pi}\left(\Phi_{1}\right) \\
\tan ^{-1} \frac{d}{c} & =\operatorname{Mod}_{2 \pi}\left(\Phi_{2}+\tan ^{-1} \frac{p_{14}}{p_{13}}\right) \\
\tan ^{-1} \frac{b}{a} & =\operatorname{Mod}_{2 \pi}\left(\Phi_{1}+\tan ^{-1} \frac{p_{32}}{p_{31}}\right) \\
\tan ^{-1} \frac{D}{C} & =\operatorname{Mod}_{2 \pi}\left(\Phi_{2}\right)
\end{aligned}
$$

where $\operatorname{Mod}_{2 \pi}$ indicates taking modulus of $2 \pi$ and we have made use of the fact that the value of arctangent can be uniquely determined within $[0,2 \pi)$ when both sine and cosine of an angle is known.

The phase advances of the normal modes $\Phi_{1,2}$ at the BPMs can be calculated with the lattice model. The $\mathbf{P}$ matrix can be computed from the one-turn transfer matrix at the BPM with the numeric procedure given in Ref. [19] or alternatively with equation $\mathbf{P}=\mathbf{V} \mathbf{U}$, with matrices $\mathbf{V}, \mathbf{U}$ calculated with formulas given in Ref. [18]. 
151 with

$$
\mathbf{B}=\left(\begin{array}{cc}
\cos \theta & \sin \theta \\
-\sin \theta & \cos \theta
\end{array}\right)\left(\begin{array}{cc}
g_{x} & 0 \\
0 & g_{y}
\end{array}\right)
$$

152 where $g_{x, y}$ and $\theta$ being the BPM gains and BPM roll, respectively. Transforma-

153 tion in Eq. (12) is applied to both the transformation matrix $\mathbf{P}$ and the model

154 dispersion functions before they are compared to the measurements. In LOCO

Eqs. (4)-(11) apply at each BPM location. They relate quantities measured from TbT BPM data to quantities that can be calculated with the lattice model. n reality, the actual machine lattice deviates from the ideal model and there are intrumentation errors in the measurements. Therefore, Eqs. (4)-(11) are not exactly satisfied. However, one could adjust parameters in the lattice model and the diagnostics to minimize the differences between the measurements and the model prediction through a least-square problem. Based on these equations, an approach to calibrate the lattice model with TbT BPM data can be developed in a manner similar to the LOCO method, except here the input data used are different.

Dispersion function measurements can be done quickly. They provide additional constraints to the lattice model and are themselves targets to be controlled through optics and coupling correction. Therefore, dispersion functions are also cluded in the least-square fitting problem.

Instrumentation errors include BPM gain errors and BPM rolls. For each $\mathrm{BPM}$, the measured beam position is related to actual beam position via a transformation

$$
\left(\begin{array}{l}
x \\
y
\end{array}\right)_{\text {meas }}=\mathbf{B}\left(\begin{array}{l}
x \\
y
\end{array}\right)_{\text {beam }},
$$
a fourth parameter is included in matrix $\mathbf{B}$ to describe BPM transformation errors [1]. We may do the same in the future in our method.

The objective function to be minimized can be defined as

$$
f(\mathbf{q})=\chi^{2}=\sum_{i, k} w_{k}\left(\frac{d_{i k}^{\text {meas }}-d_{i k}^{\text {model }}}{\sigma_{i k}}\right)^{2},
$$


where $\mathbf{q}$ is a vector that holds all fitting parameters, summation index $i$ stands for BPM $i, k$ stands for the $k^{\prime}$ th type of data, $d_{i k}^{\text {meas }}$ and $d_{i k}^{\text {model }}$ are the measured and model values for data, respectively, $\sigma_{i k}$ is the uncertainty level for data point $d_{i k}^{\text {meas }}$, and $w_{k}$ is the weight factor for data type $k$. The fitting parameters include horizontal and vertical BPM gains, BPM rolls, quadrupole gradients, and skew quadrupole gradients. For each BPM, the fitting data include the amplitude of the horizontal primary mode (Eq. (4)), the amplitude of the vertical primary mode (Eq. (6)), the amplitude of the horizontal secondary mode (Eq. (5)), the amplitude of the vertical secondary mode (Eq. (7)), horizontal dispersion, vertical dispersion, cosine and sine of the phase advance of the horizontal primary mode (Eq. (8)), cosine and sine of the phase advance of the horizontal secondary mode (Eq. (9)), cosine and sine of the phase advance of the vertical primary mode (Eq. (11)), and cosine and sine of the phase advance of the vertical secondary mode (Eq. (10)). The betatron tunes can also be included in the objective function as fitting data. The weight factors are set to 1 by default and may be empirically adjusted to change the relative weights of different types of data. More weight may be given to the phase terms as they are not affected by BPM calibration. It is also advisable not to let contribution of one type of data to dominate $\chi^{2}$.

TbT BPM data and orbit response matrix data sample the linear optics and coupling of the storage ring in a similar manner, i.e., by perturbing the beam orbit in the transverse phase space area around the closed orbit. Orbit response matrix data are measured with closed orbit, which typically has higher precision than TbT orbit because closed orbit is usually measured by averaging over many turns. For example, for the NSLS-II storage ring, the noise level for closed orbit measurement is $\sim 0.2 \mu \mathrm{m}$ and the noise level for TbT orbit is $\sim$ $1.0 \mu \mathrm{m}$. However, in TbT BPM data analysis, hundreds to thousands turns can be used, which statistically compensates the loss of precision for single-pass orbit measurements. In fact, because the advanced data analysis technique, ICA, is used to extract the betatron modes with data from all BPMs while filtering out BPM noise and contributions of other physical processes, it is possible that 
TbT BPM data have a statistical advantage over orbit response matrix data for optics and coupling measurements.

It is known that quadrupole strength fitting parameters in a storage ring lattice could have similar patterns in their perturbations to the optics and it may be difficult to separate their contributions to optics errors through fitting the orbit response matrix data [20]. The same is true to our TbT data based fitting method. Therefore, the constrained fitting technique proposed for LOCO in Ref. [20] should be used here, too. With such a fitting algorithm, the ICA based optics and coupling correction method described in the above demonstrated high robustness in both simulation and experiments.

\section{Simulation}

We have done simulation studies to demonstrate the applicability of the proposed method and the results are reported in Ref. [16]. Lattice models for the SPEAR3 and NSLS-II storage rings are used in those studies. Quadrupole and skew quadrupole errors are planted into the lattice. TbT BPM data are generated by particle tracking, adding BPM gain and roll errors, and adding BPM noise. After applying ICA and fitting as described in the previous section, the BPM gain and roll errors are accurately recovered for both lattice models. The quadrupole and skew quadrupole errors are recovered for the SPEAR3 lattice. For the NSLS-II lattice, because of the degeneracy issue, only an approximately equivalent solution is obtained for the quadrupole and skew quadrupole errors. The optics error (beta beat) and coupling (ratio of projected emittance $\epsilon_{y} / \epsilon_{x}$ ) for the fitted lattice are very close to the target lattice, which indicate the fitted lattice is a good approximation to the latter.

\section{Experiments}

\subsection{Experimental setup and example ICA modes}

We applied our ICA based method to the NSLS-II storage ring to experimentally measure and correct the storage ring optics and coupling. NSLS-II storage ring is a new third generation storage ring light source that has been 

are capable of taking turn-by-turn data [21].

Table 1: Selected Parameters of NSLS-II

\begin{tabular}{l|c|l}
\hline Parameters & Value & Unit \\
\hline Energy & 3 & $\mathrm{GeV}$ \\
Circumference & 792.0 & $\mathrm{~m}$ \\
\# of periods & 30 & \\
betatron tunes $\nu_{x, y}$ & $33.22,16.26$ & \\
RF frequency $f_{\mathrm{rf}}$ & 499.68 & $\mathrm{MHz}$ \\
Momentum compaction $\alpha_{c}$ & $0.36 \times 10^{-3}$ & \\
\hline
\end{tabular}

220

recently commissioned. A few selected parameters of the NSLS-II storage ring are shown in Table 1. The storage ring is equipped with 180 BPMs, all of which

In the experiment, errors were initially inserted to the the machine lattice by adding small random errors to quadrupole magnet strengths $\left(\sigma_{\Delta K / K} \sim 10^{-4}\right)$. Coherent motion was excited simultaneously on the horizontal and vertical planes with the pingers before TbT BPM data were taken. The oscillation amplitudes for BPMs with large beta function values were about $0.2 \mathrm{~mm}$ on the horizontal plane and about $0.3 \mathrm{~mm}$ on the vertical plane, respectively. Five TbT BPM data sets were taken for each machine condition. In addition, dispersion data were taken by shifting the rf frequency by $500 \mathrm{~Hz}$. 1024 turns of BPM data were used for the ICA analysis. Data from both planes form a $360 \times 1024$ matrix. We kept 10 leading singular value modes for ICA analysis. The first four ICA modes are the betatron modes. The fifth mode is the synchrotron mode. The sixth mode shows a slow drift on its temporal pattern and its source is unknown. The other modes are mostly within the noise level. FIG. 1 shows the amplitude (rms values for all BPMs) of all ten ICA modes and the FFT spectrum of the first six modes.

The spatial pattern of the synchrotron mode (mode 5) is proportional to dispersion and can be used to measure the horizontal and vertical dispersion functions. The energy oscillation amplitude is determined by requiring the horizontal rms dispersion to be equal to the model value, from which the energy oscillation amplitude was found to be $\delta_{m}=0.3 \times 10^{-4}$. The measured syn- 

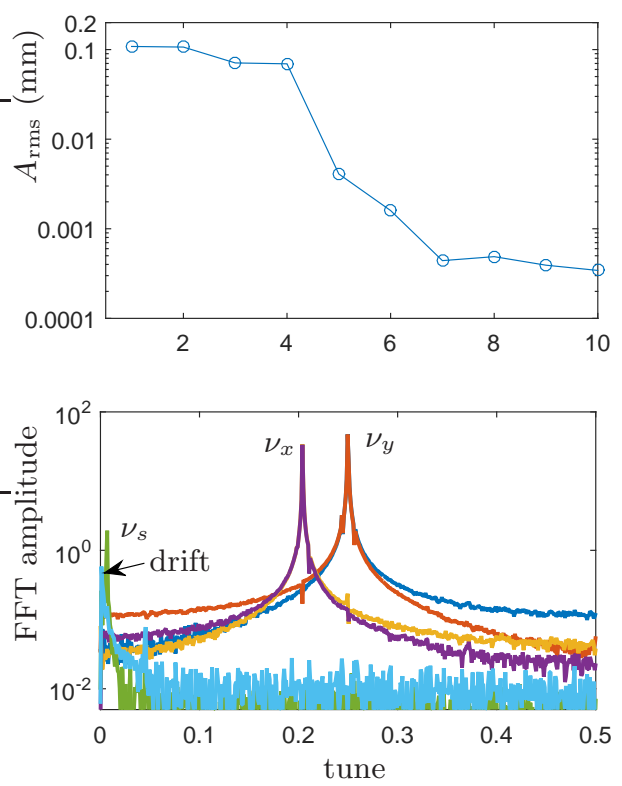

Figure 1: Rms amplitude of the first 10 leading ICA modes (top) and FFT spectrum the first six ICA modes (bottom) from the TbT BPM data of iteration 1. The FFT spectrum is scaled by the rms amplitude for each mode. 

Reasonable agreement is seen for both horizontal and vertical dispersions.

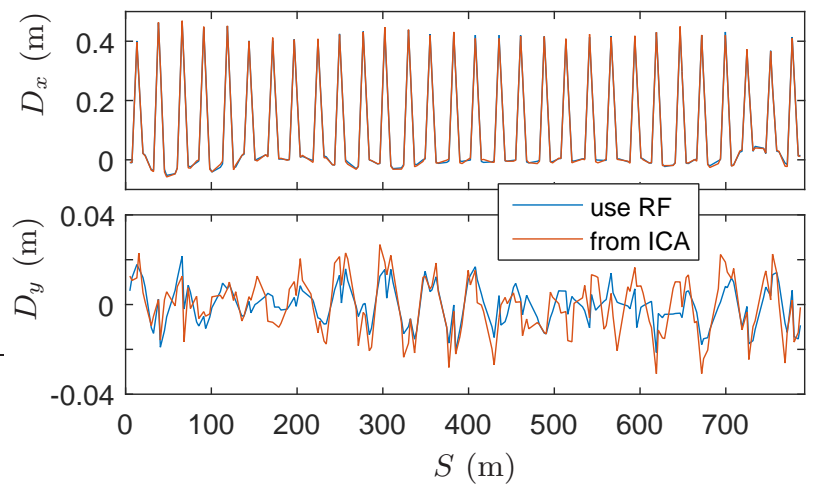

Figure 2: Horizontal (top) and vertical (bottom) dispersion functions measured with rf frequency shift ("use RF") or from the TbT data through ICA ("from ICA").

chrotron tune is $\nu_{s}=0.00657$. Dispersion functions measured from the TbT data are compared to results from the usual closed orbit based method in FIG. 2.

\subsection{Optics and coupling before correction}

From the spatial patterns of the ICA betatron modes, the betatron amplitudes and phase advances at the BPMs can be calculated [4]. By requiring the average beta functions as derived from the primary modes at the BPMs to be equal to the values of the ideal model, we can obtain the action variables of the coherent motion for the horizontal and vertical planes. For the initial data set, the action variables are $J_{1}=1.41 \times 10^{-9} \mathrm{~m}$ and $J_{2}=2.82 \times 10^{-9} \mathrm{~m}$ and the fractional betatron tunes obtained from the corresponding temporal patterns are $\nu_{x}=0.2048$ and $\nu_{y}=0.2513$, respectively. Phase advances of the normal modes can be measured from the amplitudes of the corresponding ICA modes, using Eqs. (8), (10). The measured phase advances between adjacent BPMs are compared to the model values in FIG. 3.

The ratio of the amplitudes of the projected motion of a normal mode onto the two transverse planes is a measure of the local coupling. From Eqs. (4-7), 

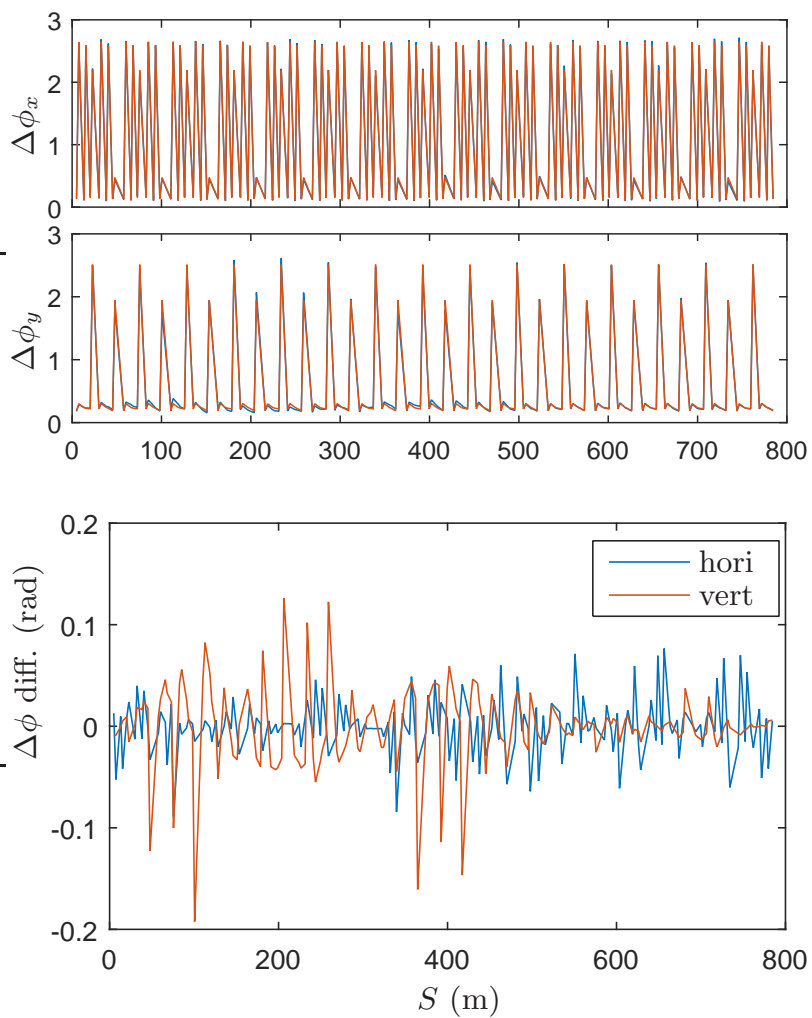

Figure 3: Top: Phase advances between adjacent BPMs from ICA measurements before corrections (blue) are compared to the ideal model (red). Bottom: Differences between measured and model phase advances between adjacent BPMs before corrections. 


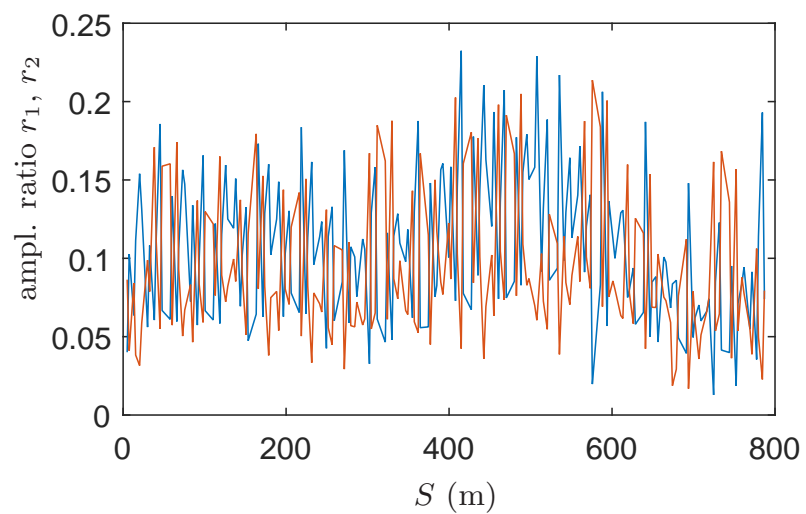

Figure 4: Amplitude ratios $r_{1}$ (blue) and $r_{2}$ (red) of the projected motion onto the two transverse planes for the two normal modes, as defined in Eq. (15), for TbT data before corrections.

we can define

$$
\begin{aligned}
& r_{1}=\frac{\sqrt{a^{2}+b^{2}}}{\sqrt{A^{2}+B^{2}}}=\frac{\sqrt{p_{31}^{2}+p_{32}^{2}}}{p_{11}}, \\
& r_{2}=\frac{\sqrt{c^{2}+d^{2}}}{\sqrt{C^{2}+D^{2}}}=\frac{\sqrt{p_{13}^{2}+p_{14}^{2}}}{p_{33}} .
\end{aligned}
$$

The amplitude ratio for the two normal modes at all BPMs are shown in FIG. 4.

The ICA results are used for lattice model fitting as described in section 2 . The fitting parameters are horizontal gains, vertical gains, and rolls for all 180 BPMs, 150 quadrupole parameters, and 30 skew quadrupole parameters. The quadrupole and skew quadrupole parameters are the same as the LOCO setup for NSLS-II [22]. The normalizing sigmas in Eq. (14) are set to $0.05 \mathrm{~m}^{-1 / 2}$ for the betatron amplitudes, $0.005 \mathrm{~m}$ for dispersion functions, $0.005 \mathrm{rad}$ for phase advances of the primary modes, and 0.02 rad for phase advances of the secondary modes. Constraints are added to all fitting parameters with the cost function proportional to the 2-norm of the corresponding column of the Jacobian matrix of each fitting parameter. $\chi^{2}$ normalized by the degree of freedom dropped from 140.9 to 1.8 in two fitting iterations for the first TbT data set.

Orbit response matrix data were also taken during the experiment under the same machine condition. The data were fitted for three iterations. The results 


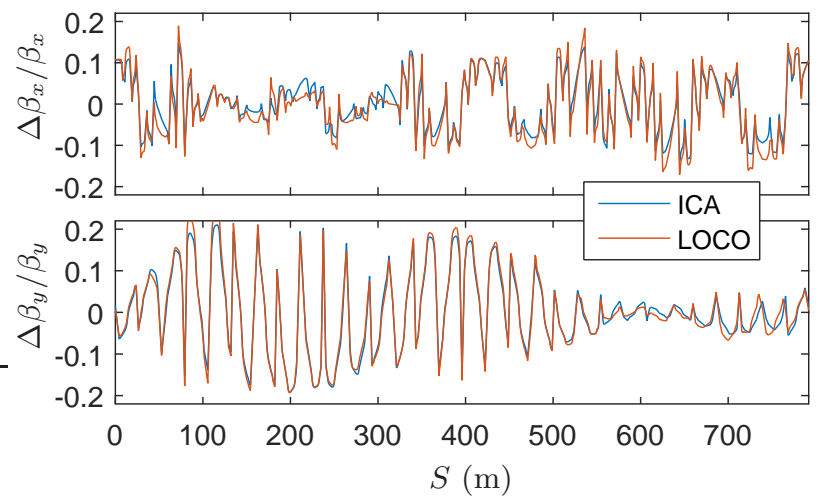

Figure 5: Comparison of beta beating of lattices fitted by TbT data ("ICA") and LOCO for horizontal (top) and vertical (bottom) beta functions before corrections.

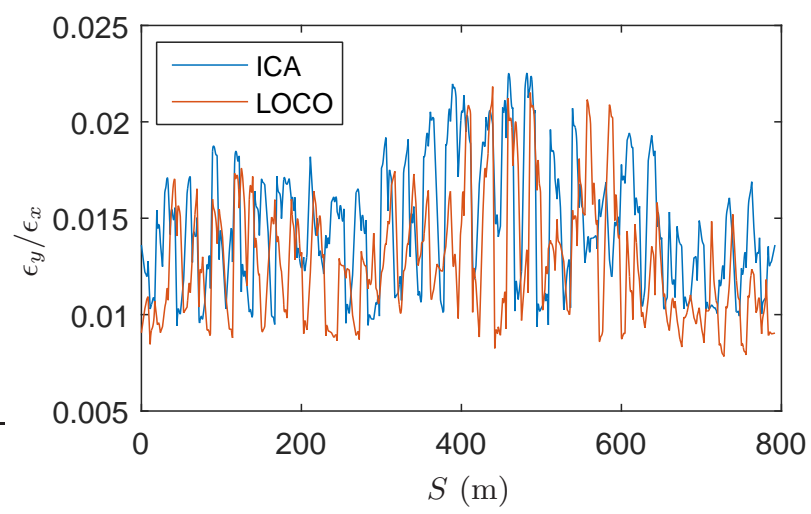

Figure 6: Comparison of ratios of projected vertical emittance over horizontal emittance for lattices fitted by TbT data ("ICA") and LOCO before corrections.

are compared to the ICA fitting results. The optics of the fitted lattices are compared in FIG. 5. There was excellent agreement between ICA and LOCO in the distribution of beta beating, which clearly verifies that the two fitted lattices are nearly equivalent. FIG. 6 shows the distribution of the projected vertical emittance (normalized by the horizontal emittance) for the two fitted latices. Although not all details agree, the average emittance ratios are nearly the same.

The fitted lattice parameters for ICA fitting and LOCO are compared in FIG. 7. Good agreement was seen for quadrupole variables 1-60 (QL1 and QL2) 

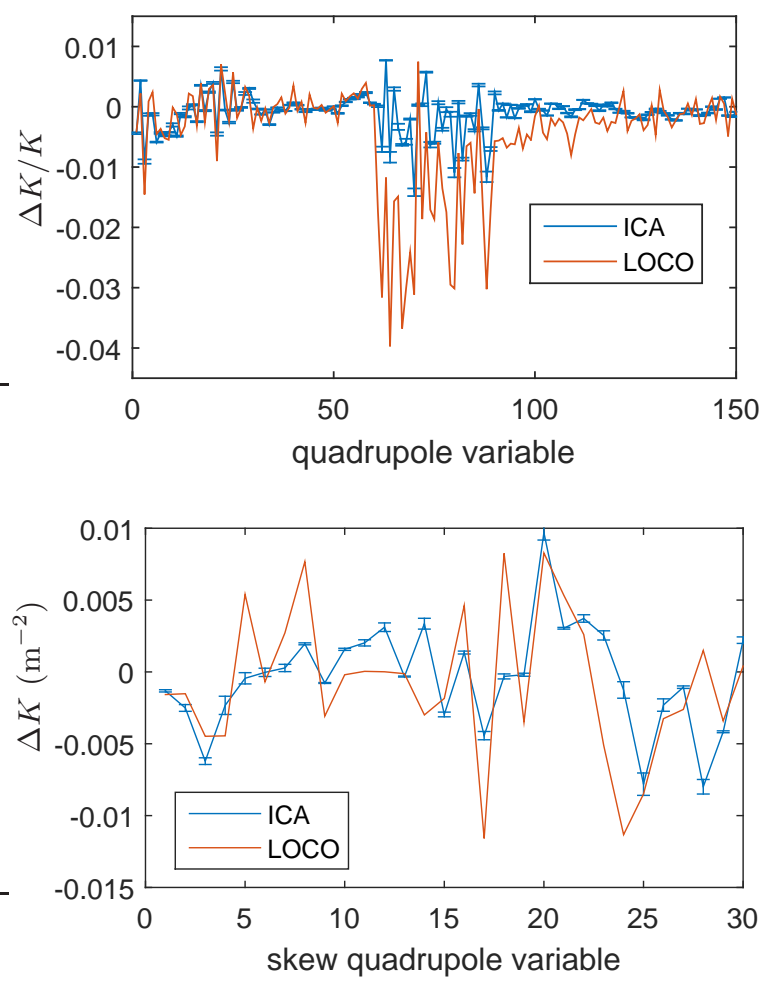

Figure 7: Comparison of fitted quadrupole variables (top) and skew quadrupole variables (bottom) for lattices fitted by TbT data ("ICA") and LOCO for iteration 1.

and 121-150 (QM2). But the fitted errors of quadrupole variables 61-120 (QH1 and $\mathrm{QH} 2$ ) for LOCO are larger. Since the fitted optics of the two methods are equivalent (see FIG.5), the differences in the fitted quadrupole errors indicate the cost functions for these variables in the LOCO setup need to be increased. The error bars for the ICA fitting method in FIG.5 are derived from the fitting results of the five TbT data sets under the same condition.

The ICA fitting results of quadrupole and skew quadrupole variables were applied to the machine to correct optics and coupling. Then a new TbT BPM data set was taken, fitted, and used for correction. A total of three corrections were applied. Final TbT BPM data sets and an orbit response matrix data set were taken after the corrections. FIG. 8 shows the initial $\chi^{2}$ (before fitting) normalized by the degree of freedom for the four TbT BPM data sets. The 
reduction of initial $\chi^{2}$ indicates that the optics and coupling were being improved through tho itnmetinna of snrenstinna

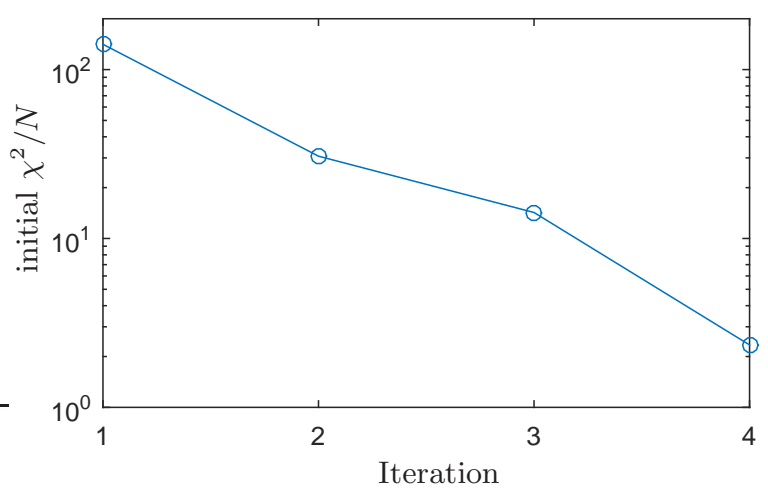

Figure 8: Initial normalized $\chi^{2}$ (before fitting) for the four TbT BPM data sets taken during the experiment.

\subsection{Optics and coupling after correction}

A series of measures directly derived from the TbT BPM data or dispersion measurements before and after the ICA fitting based corrections are shown in Table 2. After corrections, the measured fractional betatron tunes are much closer to their nominal values. The rms values of the phase advance differences between measurements and models (shown in FIG. 9) are a factor of 3.6 and 5.3 smaller from the initial values (before corrections) for the two transverse planes, respectively. The measured dispersion functions after corrections are shown in FIG. 10. Compared to measurements before corrections (in FIG. 2), significant improvements were made on both horizontal and vertical dispersions. The residual vertical dispersion includes sharp peaks that are due to rolls of BPMs at large horizontal dispersion locations.

The coupling amplitude ratios as defined in Eq.(15) after correction are shown in FIG. 11. The average ratios are reduced by about a factor of 2 . The direct measures in Table 2 clearly show that both the linear optics and linear coupling were significantly improved with the ICA fitting based corrections.

The ICA fitting results from the final TbT BPM data are also compared to the fitting results of the final LOCO data. The rms beta beating and average 

coupling ratio obtained with the two methods before and after corrections are shown in Table 3. FIG. 12 shows beta beating from the two methods after

Table 2: Direct lattice characterization before and after corrections.

\begin{tabular}{l|cc}
\hline Parameters & before & after \\
\hline fractional $\nu_{x}$ & 0.2048 & 0.2193 \\
fractional $\nu_{y}$ & 0.2513 & 0.2602 \\
$\operatorname{rms} \Delta \phi_{x}$ diff. $(\mathrm{rad})$ & 0.0280 & 0.0077 \\
$\operatorname{rms} \Delta \phi_{y}$ diff. $(\mathrm{rad})$ & 0.0390 & 0.0074 \\
$\operatorname{rms} \Delta D_{x}(\mathrm{~m})$ & 0.0171 & 0.0049 \\
$\operatorname{rms} D_{y}(\mathrm{~m})$ & 0.0089 & 0.0046 \\
$\operatorname{mean} r_{1}$ & 0.106 & 0.052 \\
mean $r_{2}$ & 0.094 & 0.046 \\
\hline
\end{tabular}

Table 3: Fitted lattice parameters by ICA and LOCO before and after corrections.

\begin{tabular}{l|cc|cc}
\hline & before & after & \\
\hline Parameters & ICA & LOCO & ICA & LOCO \\
\hline $\operatorname{rms} \Delta \beta_{x} / \beta_{x}$ & 0.0678 & 0.0780 & 0.0051 & 0.0194 \\
$\operatorname{rms} \Delta \beta_{y} / \beta_{y}$ & 0.0937 & 0.0991 & 0.0038 & 0.0110 \\
$\operatorname{rms} \Delta D_{x}$ & 0.0167 & 0.0227 & 0.0056 & 0.0045 \\
$\operatorname{rms} \Delta D_{y}$ & 0.0080 & 0.0085 & 0.0030 & 0.0046 \\
$\operatorname{mean} \epsilon_{y} / \epsilon_{x}$ & 0.0147 & 0.0129 & 0.0027 & 0.0031 \\
\hline
\end{tabular}

In FIG. 13 we show a comparison of fitted BPM gains for the two methods.

327 At many locations the fitted gains of the two methods agree. But there are 


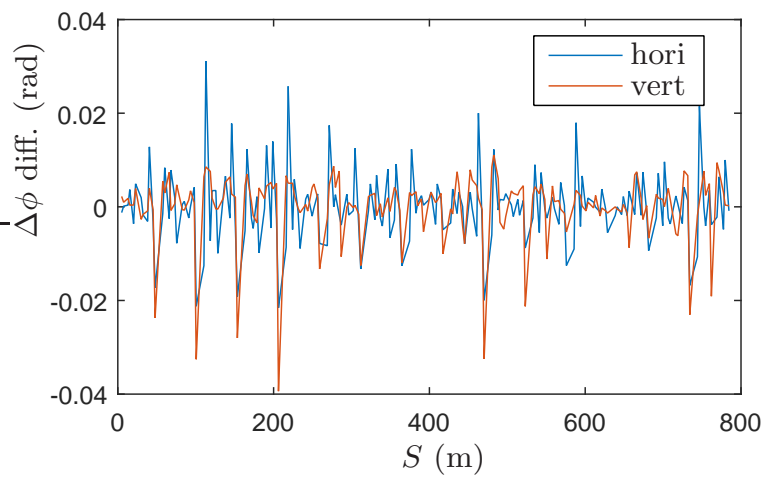

Figure 9: Differences between measured and model phase advances between adjacent BPMs after corrections. Error sigmas of primary mode phase advances derived from 5 data sets are between $0.5-2.0 \times 10^{-3}$.

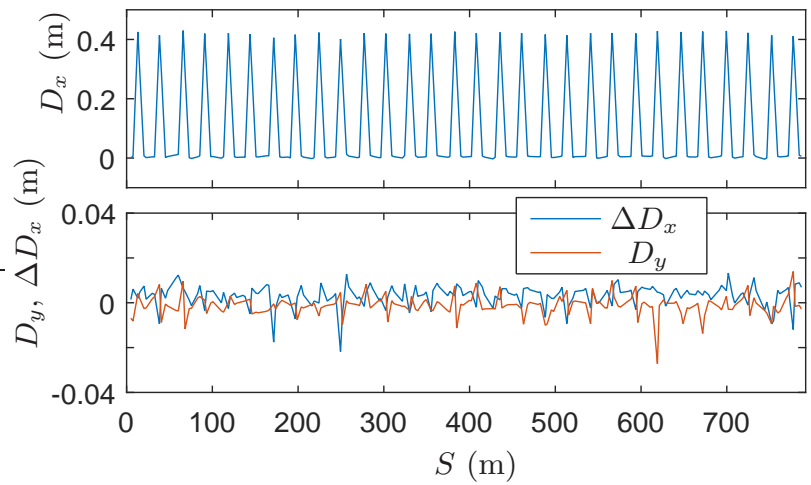

Figure 10: Measured horizontal (top) and vertical (bottom, $D_{y}$ ) dispersions and the difference of horizontal dispersion with the model dispersion (bottom, $\Delta D_{x}$ ) after corrections.

discrepancies at some locations. The BPM gains fitted with the TbT data could

be more susceptible to noise or coupling with optics errors. A good practice may be to use fixed BPM gains obtained with LOCO in the ICA fitting method.

\section{Conclusion}

We propose a method to use turn-by-turn (TbT) BPM data to simultaneously correct linear optics and linear coupling for circular accelerators. The independent component analysis (ICA) is applied to extract the betatron normal modes and their projection on the two transverse planes [4]. The betatron amplitudes and phase advances of the projected modes are fitted to the lattice 


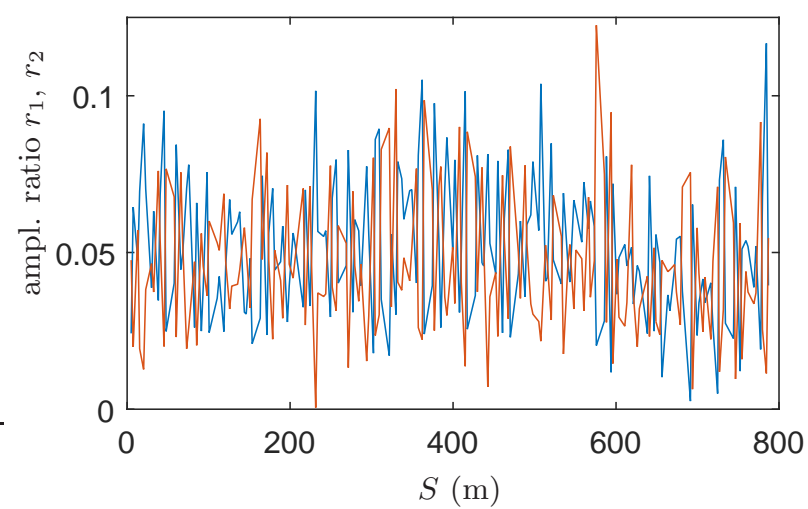

Figure 11: Amplitude ratios $r_{1}$ (blue) and $r_{2}$ (red) of the projected motion onto the two transverse planes for the two normal modes for TbT data after corrections. The average error sigmas for $r_{1}$ and $r_{2}$ are $1.2 \times 10^{-3}$ and $0.6 \times 10^{-3}$, respectively.

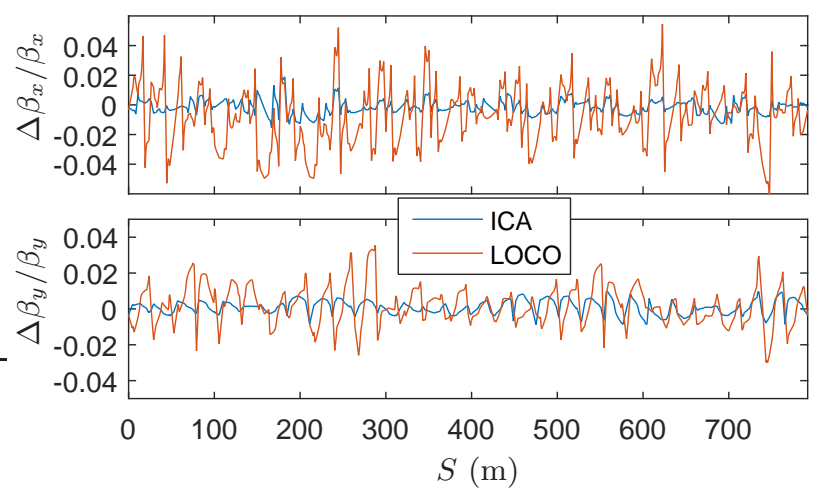

Figure 12: Comparison of beta beating of lattices fitted by TbT data ("ICA") and LOCO for horizontal (top) and vertical (bottom) beta functions after corrections. The fitted errors in LOCO is partially due to an orbit shift of up to $1 \mathrm{~mm}$ during the orbit response matrix measurement. 


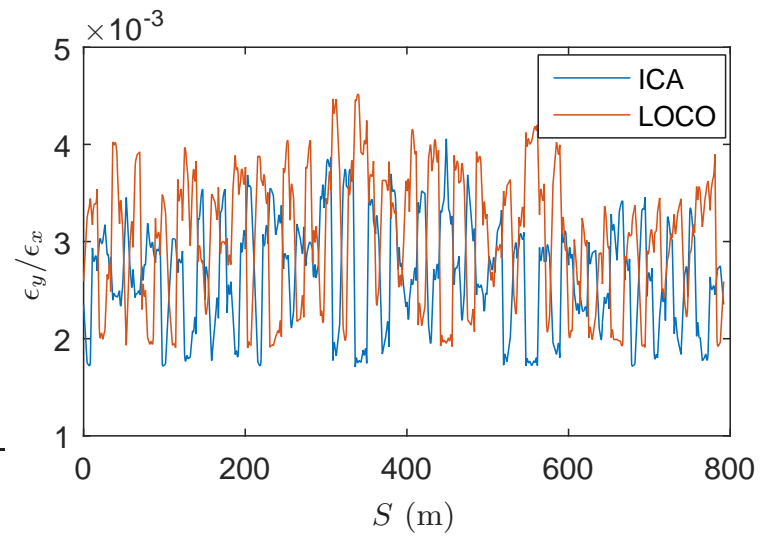

Figure 13: Comparison of ratios of projected vertical emittance over horizontal emittance for lattices fitted by TbT data ("ICA") and LOCO after corrections.

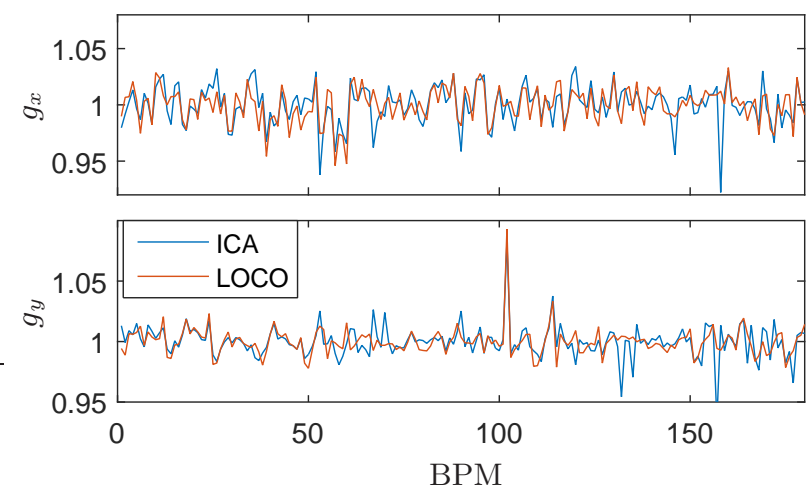

Figure 14: Comparison of fitted BPM gains by TbT data ("ICA") and LOCO for horizontal (top) and vertical (bottom) BPMs after corrections. 
model. Linear optics and coupling of the machine can be calculated with the fitted model. Correction is made by reversing the fitted errors of the quadrupole and skew quadrupole variables.

This new method has been successfully demonstrated on the NSLS-II storage ring. In the experiment we reduced rms beta beat from $7.8 \%$ (horizontal) and $9.9 \%$ (vertical) to $1.9 \%$ (horizontal) and $1.1 \%$ for the two transverse planes, respectively, using LOCO results as references. The coupling ratio was reduced from $1.3 \%$ to $0.3 \%$.

Because TbT can be taken within seconds and with little perturbation to the stored beam (in our experiment coherent oscillation amplitude is below $0.3 \mathrm{~mm}$ ), this method has a great advantage over the orbit response matrix based method, LOCO [1]. For storage ring machines, this method may be used to check and correct optics during operation using data taken with injection transients. It may also improve efficiency for machine startup and machine studies as less time is needed for lattice correction.

\section{Acknowledgment}

We thank Yongjun Li for preparing the initial condition of the machine for the experiment. We thank James Safranek for reading the manuscript. Author $\mathrm{X}$. Yang thanks Victor Smalyuk for many useful discussions. The study is supported by U.S. DOE under Contract No. DE-AC02-98CH10886 (BNL) and Contract No. DE-AC02-76SF00515 (SLAC).

[1] J. Safranek, Experimental determination of storage ring optics using orbit response measurements, Nuclear Instruments and Methods in Physics Research Section A: Accelerators, Spectrometers, Detectors and Associated Equipment 388 (12) (1997) 27 - 36. doi:http://dx.doi.org/10.1016/S01689002(97)00309-4.

URL http://www.sciencedirect.com/science/article/pii/ S0168900297003094 
[2] P. Castro, et al, , in: Proceedings of PAC93, Washington, D.C., 1993, p. 2103.

[3] C.-x. Wang, V. Sajaev, C.-Y. Yao, Phase advance and $\beta$ function measurements using model-independent analysis, Phys. Rev. ST Accel. Beams 6 (2003) 104001. doi:10.1103/PhysRevSTAB.6.104001.

URL http://link.aps.org/doi/10.1103/PhysRevSTAB.6.104001

[4] X. Huang, S. Y. Lee, E. Prebys, R. Tomlin, Application of independent component analysis to fermilab booster, Phys. Rev. ST Accel. Beams 8 (2005) 064001. doi:10.1103/PhysRevSTAB.8.064001.

URL http://link.aps.org/doi/10.1103/PhysRevSTAB.8.064001

[5] M. Aiba, S. Fartoukh, A. Franchi, M. Giovannozzi, V. Kain, M. Lamont, R. Tomás, G. Vanbavinckhove, J. Wenninger, F. Zimmermann, R. Calaga, A. Morita, First $\beta$-beating measurement and optics analysis for the cern large hadron collider, Phys. Rev. ST Accel. Beams 12 (2009) 081002. doi:10.1103/PhysRevSTAB.12.081002.

URL http://link.aps.org/doi/10.1103/PhysRevSTAB.12.081002

[6] X. Shen, S. Y. Lee, M. Bai, S. White, G. Robert-Demolaize, Y. Luo, A. Marusic, R. Tomás, Application of independent component analysis to ac dipole based optics measurement and correction at the relativistic heavy ion collider, Phys. Rev. ST Accel. Beams 16 (2013) 111001. doi:10.1103/PhysRevSTAB.16.111001. URL http://link.aps.org/doi/10.1103/PhysRevSTAB.16.111001

[7] M. Aiba, M. Böge, J. Chrin, N. Milas, T. Schilcher, A. Streun, Comparison of linear optics measurement and correction methods at the swiss light source, Phys. Rev. ST Accel. Beams 16 (2013) 012802. doi:10.1103/PhysRevSTAB.16.012802.

URL http://link.aps.org/doi/10.1103/PhysRevSTAB.16.012802

[8] M. Carla, et al, , in: Proceedings of IPAC2015, Richmond, VA, USA, 2015, p. 1686. 
[9] J. Bengtsson, CERN 88-05 (1988).

[10] R. Tomás, , Ph.D. thesis, University of Valencia (Jan 2003).

[11] M. Benedikt, F. Schmidt, R. Tomás, P. Urschütz, A. Faus-Golfe, Driving term experiments at cern, Phys. Rev. ST Accel. Beams 10 (2007) 034002. doi:10.1103/PhysRevSTAB.10.034002. URL http://link.aps.org/doi/10.1103/PhysRevSTAB.10.034002

[12] F. Wang, S. Y. Lee, Vertical-beam emittance correction with independent component analysis method, Phys. Rev. ST Accel. Beams 11 (2008) 050701. doi:10.1103/PhysRevSTAB.11.050701.

URL http://link.aps.org/doi/10.1103/PhysRevSTAB.11.050701

[13] A. Franchi, L. Farvacque, J. Chavanne, F. Ewald, B. Nash, K. Scheidt, R. Tomás, Vertical emittance reduction and preservation in electron storage rings via resonance driving terms correction, Phys. Rev. ST Accel. Beams 14 (2011) 034002. doi:10.1103/PhysRevSTAB.14.034002.

URL http://link.aps.org/doi/10.1103/PhysRevSTAB.14.034002

[14] X. Huang, J. Sebek, D. Martin, Lattice modeling and calibration with turn-by-turn orbit data, Phys. Rev. ST Accel. Beams 13 (2010) 114002. doi:10.1103/PhysRevSTAB.13.114002.

URL http://link.aps.org/doi/10.1103/PhysRevSTAB.13.114002

[15] R. Tomás, O. Brüning, M. Giovannozzi, P. Hagen, M. Lamont, F. Schmidt, G. Vanbavinckhove, M. Aiba, R. Calaga, R. Miyamoto, Cern large hadron collider optics model, measurements, and corrections, Phys. Rev. ST Accel. Beams 13 (2010) 121004. doi:10.1103/PhysRevSTAB.13.121004. URL http://link.aps.org/doi/10.1103/PhysRevSTAB.13.121004

[16] X. Huang, X. Yang, Linear Optics and Coupling Correction with Turnby-turn BPM Data, in: Proceedings of IPAC15, Richmond, VA, 2015, p. 698. 
[17] D. Edwards, L. Teng, IEEE Trans. Nucl. Sci. 20 (1973) 3.

[18] D. Sagan, D. Rubin, Linear analysis of coupled lattices, Phys. Rev. ST Accel. Beams 2 (1999) 074001. doi:10.1103/PhysRevSTAB.2.074001.

URL http://link.aps.org/doi/10.1103/PhysRevSTAB.2.074001

[19] Y. Luo, Linear coupling parametrization in the actionangle frame, Phys. Rev. ST Accel. Beams 7 (2004) 124001. doi:10.1103/PhysRevSTAB.7.124001.

URL http://link.aps.org/doi/10.1103/PhysRevSTAB.7.124001

[20] X. Huang, J. Safranek, G. Portmann, Loco with constraints and improved fitting technique, ICFA Newsletter 44 (2007) 60.

URL http://www.slac.stanford.edu/cgi-wrap/getdoc/ slac-pub-13686.pdf

[21] K. Vetter, et al., NSLS-II RF beam position monitor, in: Proceedings of BIW10, Santa Fe, NM, 2010, p. 205.

[22] X. Yang, X. Huang, J. Safranek, Loco application to NSLS2 storage ring dispersion and beta beating correction, in: Proceedings of IPAC15, Richmond, VA, 2015, p. 1989. 